\title{
The Effect of Binary Interactions in Infrared Passbands
}

\author{
F. Zhang, L. Li and Z. Han \\ National Astronomical Observatories/Yunnan Observatory, Chinese Academy of Sciences, \\ PO Box 110, Kunming, Yunnan Province 650011, China \\ email: gssephd@public.km.yn.cn or zhangfh@ynao.ac.cn
}

\begin{abstract}
We present the integrated $J, H, K, L, M$ and $N$ magnitudes and the colours involving infrared bands, for an extensive set of instantaneous-burst binary stellar populations (BSPs) by using evolutionary population synthesis (EPS). By comparing the results for BSPs WITH and WITHOUT binary interactions we show that the inclusion of binary interactions makes the magnitudes of populations larger (fainter) and the integrated colours smaller (bluer) for $\tau \geqslant 1$ Gyr. Also, we compare our model magnitudes and colours with those of Bruzual \& Charlot (2003, hereafter BC03) and Maraston (2005, hereafter M05). At last, we compare these model broad colours with Magellanic Clouds globular clusters (GCs) and Milky Way GCs. In $(V-R)-[\mathrm{Fe} / \mathrm{H}]$ and $(V-I)-[\mathrm{Fe} / \mathrm{H}]$ diagrams it seems that our models match the observations better than those of $\mathrm{BC} 03$ and $\mathrm{M} 05$.
\end{abstract}

Keywords. infrared: general, binaries: general, stars: evolution, galaxies: clusters: general.

Introduction In previous paper (Zhang et al. 2005) we took into account binary interactions (BIs) in evolutionary population synthesis (EPS) models, presented the integrated $U-B, B-V, V-R$ and $V-I$ colours of binary stellar populations (BSPs), while did not give the infrared magnitudes and colours because larger fluctuations exist. However, these results in infrared passbands are very important in EPS models because the infrared light can reflect the metallicity of populations and the visible/infrared colours are the candidates of breaking the degeneration between age and metallicity.

Results We present the infrared integrated magnitudes and colours for BSPs. The ages of BSPs are in the range 1-15 Gyr, the metallicities are in the range 0.0001-0.03.

In Fig. 1 we present the bolometric magnitude $M_{\mathrm{BOL}}, K$ magnitude, $B-V$ and $V-K$ colours at $Z=0.02,0.004$ and 0.0001 for BSPs WITH and WITHOUT BIs, the results of Bruzual \& Charlot (2003, hereafter BC03) using Salpeter (1955, hereafter BC03-S) and Chabrier (2003, hereafter BC03-C) IMFs, the results of Maraston (2005, hereafter M05) using Salpeter (1955, hereafter M05-S) and Kroupa (2001, hereafter M05-K) IMFs, and the recent $V-K$ of Bruzual (2007, hereafter B07) at solar metallicity. By comparison we see that (i) the magnitudes and colours of BSPs WITH BIs are greater (fainter) and smaller (bluer) than those WITHOUT BIs, respectively. (ii) The magnitudes of BC03-S, BC03-C, M05-S and M05-K are greater than ours. (iii) The shape of the evolutionary curves of these colours is significantly different. In Fig. 2 we compare the model colours with Magellanic Clouds globular clusters (GCs) with the type of Searle, Wilkinson \& Bagnuolo(1980, hereafter SWB) in the range of 3-7 and the young star clusters in the merger remnant galaxy NGC 7252 in $(B-V)$.vs. $(U-B)$ and $(B-V)$.vs. $(V-K)$ diagrams. It shows that the $\mathrm{BC} 03$ and our models agree with the observations in $(B-V)$.vs. $(U-B)$ diagram; while in $(B-V)$.vs. $(V-K)$ diagram larger discrepancies exist among models. In Fig. 3 we compare the model broad colours with Milky Way GCs in colour-metallicity 

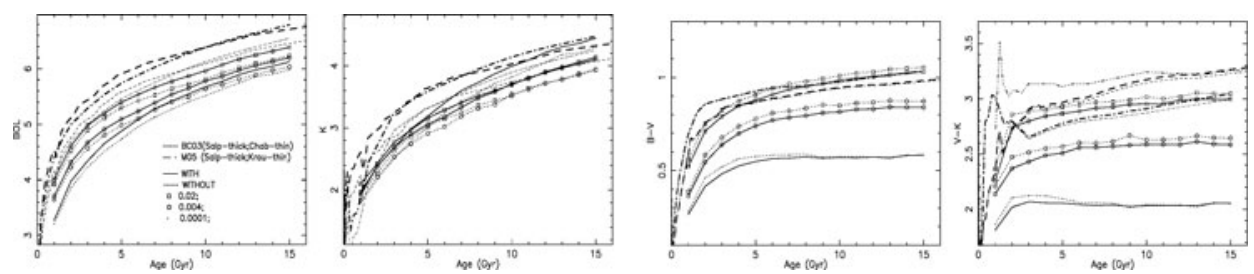

Figure 1. The bolometric magnitude $\left(M_{\mathrm{BOL}}\right), K$ magnitude, $B-V$ and $V-K$ colours for BSPs WITH and WITHOUT BIs at $Z=0.02,0.04$ and 0.0001 . Also shown are the results of BC03-S, BC03-C, M05-S and M05-K at solar metallicity. In $V-K$ diagram the recent results of B07 are shown (dash-dot-dot-dot).
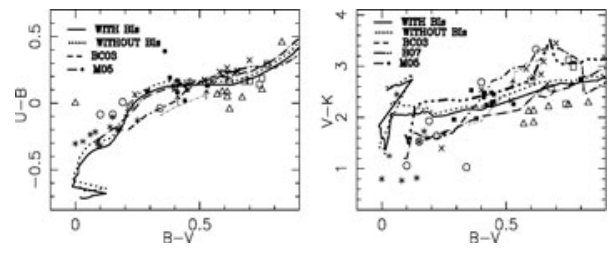

Figure 2. $B-V$ versus $U-B, B-V$ versus $V-K$ colours of star clusters. The different symbols represent Magellanic Clouds GCs with the SWB type in the range 3-7. Solid rectangles show the young star clusters in the merger remnant galaxy NGC 7252. The full and dotted lines show the evolution of BSPs WITH and WITHOUT BIs at $Z=0.01$, the ages of BSPs are greater than a few Myr. The dashed lines are the BC03-S (thick) and BC03-C (thin) models at $Z=0.008$, the dot-dash lines are the M05-S (thick) and M05-K (thin) models at $Z=0.01$ with age $\log \tau>8 \mathrm{yr}$, respectively. In $V-K$ diagram the values of B07 at solar metallicity are also shown (dash-dot-dot-dot).
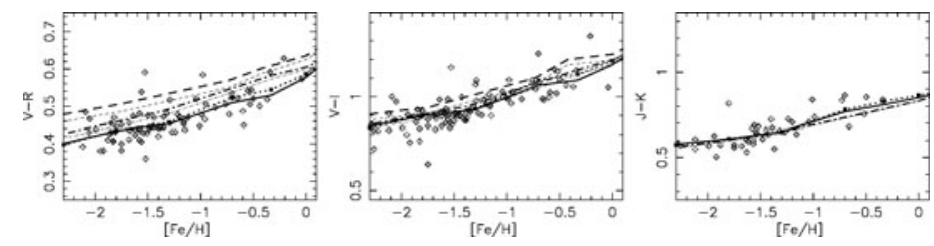

Figure 3. Comparison of colours with data of Milky Way GCs. The lines represent the same models as in Fig. 2. All models have the same age $\tau=13 \mathrm{Gyr}$

diagrams. It shows that our models match the observations better than M05 and BC03 in $(V-R)-[\mathrm{Fe} / \mathrm{H}]$ and $(V-I)-[\mathrm{Fe} / \mathrm{H}]$ diagrams.

Acknowledgments This work was funded by the Chinese Natural Science Foundation (Grant Nos 10773026, 10673029, 10433030 \& 10521001) and by Yunnan Natural Science Foundation (Grant Nos 2005A0035Q \& 2007A113M).

\section{References}

Bruzual, G. A. 2007, IAUs, Stellar populations as Building Blocks of Galaxies 241, 125 (B07) Bruzual, G. A. \& Charlot, S. 2003, MNRAS 344, 1000 (BC03)

Chabrier, G. 2003, PASP 115, 763

Kroupa, P. 2001, MNRAS 322, 231

Maraston, C. 2005, MNRAS 362, 799 (M05)

Salpeter, E. E. 1955, ApJ 121, 161

Searale, L., Wilkinson, A., \& Bagnuolo, W. G. 1980, ApJ 239, 803

Zhang, F., Han, Z., Li, L., \& Hurley, J. R. 2005, MNRAS 357, 1088 